\title{
Disk-Shaped Cobalt Nanocrystals as Fischer-Tropsch Synthesis Catalysts Under Industrially Relevant Conditions
}

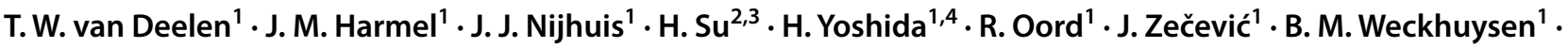 \\ K. P. de Jong ${ }^{1}$
}

Published online: 6 May 2020

(c) The Author(s) 2020

\begin{abstract}
Colloidal synthesis of metal nanocrystals (NC) offers control over size, crystal structure and shape of nanoparticles, making it a promising method to synthesize model catalysts to investigate structure-performance relationships. Here, we investigated the synthesis of disk-shaped Co-NC, their deposition on a support and performance in the Fischer-Tropsch (FT) synthesis under industrially relevant conditions. From the NC synthesis, either spheres only or a mixture of disk-shaped and spherical Co-NC was obtained. The disks had an average diameter of $15 \mathrm{~nm}$, a thickness of $4 \mathrm{~nm}$ and consisted of hcp Co exposing (0001) on the base planes. The spheres were $11 \mathrm{~nm}$ on average and consisted of $\varepsilon$-Co. After mild oxidation, the CoO-NC were deposited on $\mathrm{SiO}_{2}$ with numerically $66 \%$ of the $\mathrm{NC}$ being disk-shaped. After reduction, the catalyst with spherical plus disk-shaped Co-NC had $50 \%$ lower intrinsic activity for FT synthesis $\left(20\right.$ bar, $\left.220{ }^{\circ} \mathrm{C}, \mathrm{H}_{2} / \mathrm{CO}=2 \mathrm{v} / \mathrm{v}\right)$ than the catalyst with spherical NC only, while $\mathrm{C}_{5+}$-selectivity was similar. Surprisingly, the Co-NC morphology was unchanged after catalysis. Using XPS it was established that nitrogen-containing ligands were largely removed and in situ XRD revealed that both catalysts consisted of $65 \%$ hcp Co and 21 or $32 \%$ fcc Co during FT. Furthermore, 3-5 nm polycrystalline domains were observed. Through exclusion of several phenomena, we tentatively conclude that the high fraction of (0001) facets in diskshaped Co-NC decrease FT activity and, although very challenging to pursue, that metal nanoparticle shape effects can be studied at industrially relevant conditions.
\end{abstract}

Keywords Cobalt $\cdot$ Nanocrystals $\cdot$ Anisotropy $\cdot$ Disk $\cdot$ Model catalyst $\cdot$ Fischer-Tropsch synthesis

\section{Introduction}

Electronic supplementary material The online version of this article (https://doi.org/10.1007/s11244-020-01270-7) contains supplementary material, which is available to authorized users.

K. P. de Jong

k.p.dejong@uu.nl

1 Inorganic Chemistry and Catalysis, Debye Institute for Nanomaterials Science, Utrecht University,

Universiteitsweg 99, 3584 CG Utrecht, The Netherlands

2 Laboratory of Materials and Interface Chemistry \& Center for Multiscale Electron Microscopy, Department of Chemical Engineering and Chemistry, Eindhoven University of Technology, PO box 513, 5600 MB Eindhoven, The Netherlands

3 Institute of Complex Molecular Systems, Eindhoven University of Technology, PO box 513, 5600 MB Eindhoven, The Netherlands

4 The Institute of Scientific and Industrial Research, Osaka University, 8-1 Mihogaoka, Ibaraki, Osaka 567-0047, Japan
Controlled synthesis of supported metal nanoparticles is one of the main challenges in designing heterogenous catalysts for major industrial processes as well as fundamental research [1-3]. Traditional catalyst synthesis methods have in common that nanoparticle formation takes place on the support [4]. Recent advances in catalyst synthesis include the use of colloidal techniques to synthesize nanocrystals (NC) in solution followed by deposition on a support $[4,5]$. By separating the $\mathrm{NC}$ synthesis from deposition onto the support, the properties of the $\mathrm{NC}$ can be manipulated with greater precision, leading for example to $\mathrm{NC}$ of particular sizes, crystal structures and shapes [6]. Among these properties, nanoparticle shape is arguably the hardest to tune using conventional techniques, so here colloidal methods could offer a clear advantage over existing catalyst synthesis techniques, provided that challenges regarding NC deposition onto the support and ligand removal are solved [7]. 
Anisotropic NC are often metastable compared to spherical NC, which are usually the equilibrium shape in particular in the case of metals $[8,9]$. By using ligands that bind preferentially to specific surface facets, it is possible to inhibit growth in certain directions and to drive the process towards a kinetically controlled product $[10,11]$. Distinct crystal facets are exposed at the surface of these particles, which may lead to distinct catalytic performance. Additionally, different shapes also imply different relative amounts of corner, edge and terrace sites, the reactivity of which can vary depending on the type of reaction [12]. The effect of NC shape on catalytic performance has already been investigated mainly for noble metal [13-15] or for metal oxide NC [16], yet few accounts of shape variation for supported base metal NC catalysts exist.

The shape of NC plays an important role if the catalyzed reaction is structure sensitive. One pronounced example of such a reaction is the Fischer-Tropsch (FT) synthesis [12]. In FT, a mixture of $\mathrm{H}_{2}$ and $\mathrm{CO}$ called synthesis gas is converted into hydrocarbon fuels and chemicals over a catalyst, typically based on Fe or Co [17]. The cobalt-catalyzed FT reaction is sensitive to various structural aspects of the Co particles [18]. For example, a size dependency of the catalytic performance of cobalt is generally reported, with the surface-specific activity and $\mathrm{C}_{5+}$-selectivity decreasing sharply below a particle size of 6-8 $\mathrm{nm}$ [19-21]. Additionally, several studies indicated that the crystal structure of Co is relevant, with hcp being more active than fcc [22-26], although this topic remains controversial [27]. Given the potential influence of the crystal structure, the shape of Co particles might be important as well, as shape partially governs the surface structure of nanoparticles.

For the potential effect of the NC shape on catalytic FT performance we should resort mainly to theoretical and surface science studies, as little experimental data is available on complex model catalysts under high-pressure conditions. Theoretical studies predict that the B5 surface ensemble is essential for CO-dissociation, which is considered an important step for activity and selectivity. The occurrence of coordinatively unsaturated sites, including B5 sites, varies with particle size [28, 29] and crystal structure [29] and offers one explanation for the observed structure sensitivity. Furthermore, a computational comparison of the FT activity of various Co crystal planes indicated that higher index planes of hcp Co are orders of magnitude more active than the close packed (0001) [25]. Surface science experiments on single crystal planes of hcp Co showed that relatively more $\mathrm{CH}_{4}$ is produced on hcp (0001) than on higher index planes, leading to a lower selectivity towards $\mathrm{C}_{5+}$-products $[30,31]$. However, a recent experimental study indicated that hcp (0001) displayed intermediate activity and $\mathrm{C}_{5+}$-selectivity, with both increasing in the order hep $\{11 \overline{2} 0\}<\{0001\}<\{10 \overline{1} 1\}$ [32]. All of these aspects of surface composition could in principle be affected by the shape of the Co-NC, although it is also possible that surface defects [33], hydrocarbon coverage [34] or CO-induced surface reconstruction [35-41] might in practice annihilate the effects of Co-NC shape.

Surface science cannot always be directly translated to more complex catalysts and high-pressure systems [5]. To bridge the gap between surface science and supported catalysts under industrially relevant reaction conditions, 3D model catalysts containing anisotropic Co particles are required. Examples of anisotropic cobalt nanoparticles in FT include unsupported particles, such as $\mathrm{CoMnO}_{\mathrm{x}}$ nanoprisms [42], Co nanocubes [43], bimetallic nanorods [44], and $\mathrm{Co}_{3} \mathrm{O}_{4}$ nanorods [45], plates or cubes [32]. Furthermore, hcp nanorods supported either on $\mathrm{SiO}_{2}-\mathrm{Al}_{2} \mathrm{O}_{3}[46]$ or on $\mathrm{Ni}$ or $\mathrm{Cu}$ foams [47] have also been employed. Clearly, synthetic approaches are still required to produce anisotropic model systems that closely resemble conventional supported cobalt catalyst, with stable Co-NC shapes yet without additives, such as a second metal, that might influence the FT performance.

Here, we report our efforts to advance the synthesis, characterization and testing of shape-controlled Co-NC dispersed on a support as relevant model catalysts for the Fischer-Tropsch synthesis. We consistently obtained a mixture of disk-shaped and spherical Co-NC. The disks were $15 \mathrm{~nm}$ in diameter with a thickness of $4 \mathrm{~nm}$ and consisted of hcp Co with (0001) terminating the base planes, while the spherical particles consisted of $\varepsilon$-Co. After low-temperature oxidation of the Co-NC, the $\mathrm{CoO}-\mathrm{NC}$ were deposited on $\mathrm{SiO}_{2}\left(50 \mathrm{~m}^{2} \mathrm{~g}^{-1}\right)$. Numerically $66 \%$ of the NC were disks, leading to a higher occurrence of hcp (0001) on the cobalt surface compared to purely spherical Co-NC. The FT activity of the disks/spheres mixture was half that of spheres only, while the $\mathrm{C}_{5+}$-selectivity was identical. Remarkably, the shape endured the FT conditions, as $62 \%$ of the NC after catalysis were disks of the original dimensions. Nitrogencontaining ligands were largely removed, and both catalysts featured the same crystalline composition, leaving the possibility for enhanced hcp (0001) exposure in disks. Based on these experiments, it was concluded that the disk shape of Co-NC had a strong influence on the FT performance, most probably caused by the introduction of a high number inactive low-index Co planes.

\section{Experimental Methods}

\subsection{Synthesis of Disk-Shaped NC}

A mixture of disk-shaped and spherical Co-NC was synthesized based on an adapted procedure originally developed by Puntes et al. [10, 48]. The ligands, $50 \mathrm{mg}$ oleic acid $(90 \%$ technical grade, Sigma-Aldrich) and $600 \mathrm{mg}$ octadecylamine 
(>99.0\% (GC), Sigma-Aldrich), were transferred to a threenecked $100 \mathrm{~mL}$ flask with two septa and a reflux condenser connected to a Schlenk line. The mixture was heated to $80{ }^{\circ} \mathrm{C}$ under vacuum using a heating mantle while magnetically stirring at $600 \mathrm{rpm}$. After $30 \mathrm{~min}$, the setup was flushed three times with $\mathrm{N}_{2}$ before switching to $\mathrm{N}_{2}$ for the remainder of the synthesis. Then, $7.5 \mathrm{~mL} \mathrm{1,2-dichlorobenzene} \mathrm{(99 \%}$ Anhydrous, Sigma-Aldrich) was added to the mixture without air-exposure, a thermocouple was inserted and the mixture was heated to reflux temperature $\left(188^{\circ} \mathrm{C}\right)$. Meanwhile, $270 \mathrm{mg}$ dicobalt octacarbonyl (95\%, stabilized with hexane, Acros Organics) was transferred to a vial in an $\mathrm{N}_{2}$-glovebox and the vial was left open inside the glovebox for $40 \mathrm{~min}$ to age. After ageing, the $\mathrm{Co}_{2}(\mathrm{CO})_{8}$ was dissolved in $1.5 \mathrm{~mL}$ 1,2-dichlorobenzene and the vial was closed using a cap with a septum and removed from the glovebox. Once the liquid was at reflux temperature, the cobalt precursor was rapidly injected into the center of the flask using a syringe with a $\varnothing 1.2 \times 40 \mathrm{~mm}$ needle under $600 \mathrm{rpm}$ stirring. The synthesis was quenched after $5 \mathrm{~min}$ using a water bath. The water bath was removed after $\sim 3 \mathrm{~min}$ when the temperature of the reaction mixture was below $30^{\circ} \mathrm{C}$. Simultaneously, the stirring was stopped and the stirring plate placed $5 \mathrm{~cm}$ below the flask to magnetically separate the reaction product. The top $7 \mathrm{~mL}$ contained the highest concentration of disk-shaped Co-NC and was transferred to a glass centrifuge tube after $5 \mathrm{~min}$ and exposed to air from this point on. Consequently, the $\mathrm{NC}$ were oxidized to $\mathrm{CoO}$ to facilitate their uniform distribution over a support at a later stage [7]. The centrifuge tube was filled to $20 \mathrm{~mL}$ with 2-propanol ( $\geq 99.5 \%$ (GC), Merck) and centrifuged at $2500 \mathrm{G}$ for $10 \mathrm{~min}$. The purple/ brown supernatant was decanted and the residue redispersed in $0.5 \mathrm{~mL}$-hexane (99\%, Acros Organics) using sonication. Another precipitation-redispersion cycle was performed by filling the tube to $20 \mathrm{~mL}$ with 2-propanol, centrifuging at $2500 \mathrm{G}$ for $40 \mathrm{~min}$, decanting the supernatant and finally redispersing the residue in $10 \mathrm{~mL}$-hexane using sonication.

\subsection{Deposition of Disk-Shaped NC}

The disk-shaped $\mathrm{CoO}-\mathrm{NC}$ were deposited on $\mathrm{SiO}_{2}$ (Aerosil OX 50, Evonik) within $4 \mathrm{~h}$ after synthesis. To obtain enough material with sufficient metal loading, two NC synthesis batches were deposited consecutively on the same batch of support. A glass round bottom vessel with an overhead mechanical stirrer was placed in a sonication bath. $300 \mathrm{mg}$ $\mathrm{SiO}_{2}$ and $20 \mathrm{~mL}$-hexane were added and the top of the vessel was loosely covered with aluminum foil. Stirring and sonication were applied to suspend the $\mathrm{SiO}_{2}$ for $20 \mathrm{~min}$ and then the first $\mathrm{CoO}-\mathrm{NC}$ batch in $10 \mathrm{~mL}$-hexane was added dropwise in 15 min under continued stirring and sonication. The mixture was left for $45 \mathrm{~min}$ after the addition and then the $\mathrm{n}$-hexane was slowly evaporated in $15 \mathrm{~min}$ by a applying a flow of $\mathrm{N}_{2}$ through the vessel, all under sonication and stirring. This entire procedure was repeated a second time by suspending the dried uniformly grey powder again in $20 \mathrm{~mL}$ $\mathrm{n}$-hexane and adding the second batch of CoO-NC. Afterwards, the dried powder was suspended in $10 \mathrm{~mL}$-hexane and transferred to a glass centrifuge tube. The solid was precipitated by centrifuging at $2500 \mathrm{G}$ for $1 \mathrm{~min}$ and the supernatant was decanted. The residue was 5 times resuspended in $5 \mathrm{~mL}$-hexane and precipitated by centrifuging at $2500 \mathrm{G}$ for $1 \mathrm{~min}$ and finally dried at ambient temperature under vacuum for $16 \mathrm{~h}$. The dry powder was pressed and sieved to a grain size of $75-150 \mu \mathrm{m}$ (100 mg was obtained).

\subsection{Synthesis of Spherical NC}

Exclusively spherical Co-NC were synthesized according to a comparable hot-injection method, however, only oleic acid was used as ligand according to a procedure described before [49]. The synthesis was performed in $\mathrm{N}_{2}$ atmosphere using a Schlenk line. $65 \mathrm{mg}$ oleic acid was degassed under vacuum at $100{ }^{\circ} \mathrm{C}$ for $30 \mathrm{~min}$ under $650 \mathrm{rpm}$ magnetic stirring in a 3 -necked $100 \mathrm{~mL}$ flask with a condenser and two septa. The flask was flushed with $\mathrm{N}_{2}$ and the synthesis was continued under $\mathrm{N}_{2}$. Then, $7.5 \mathrm{~mL}$ 1,2-dichlorobenzene was added under $\mathrm{N}_{2}$ atmosphere and the solution was heated to $165^{\circ} \mathrm{C}$. Meanwhile, $270 \mathrm{mg}$ dicobalt octacarbonyl was dissolved in $1.5 \mathrm{~mL}$ 1,2-dichlorobenze and sealed under $\mathrm{N}_{2}$ atmosphere inside a glovebox. This cobalt precursor was then rapidly injected (needle: $\varnothing 0.9 \times 70 \mathrm{~mm}$ ) into the pre-heated solution at $750 \mathrm{rpm}$ stirring and reacted vigorously. After $20 \mathrm{~min}$, the reaction was quenched and cooled to at ambient temperature using a water bath. One septum was then removed to oxidize the $\mathrm{Co}-\mathrm{NC}$ to $\mathrm{CoO}-\mathrm{NC}$ by air-exposure at low temperature for $1 \mathrm{~h}$ (650 rpm stirring). The suspension was divided after over two glass centrifuge tubes, which were filled further to $20 \mathrm{~mL}$ with 2-propanol and centrifuged at $2200 \mathrm{G}$ for $30 \mathrm{~min}$ to precipitate the NC. The supernatant was decanted, the precipitate redispersed in $0.5 \mathrm{~mL} \mathrm{n}$-hexane by sonication and the tubes were filled again to $20 \mathrm{~mL}$ with 2-propanol and centrifuged at $2200 \mathrm{G}$ for $40 \mathrm{~min}$. This precipitationredispersion cycle was repeated two more times and afterwards, the residue was redispersed and combined in $2 \mathrm{~mL}$ n-hexane in total.

\subsection{Deposition of Spherical NC}

The spherical CoO-NC were deposited on the same low surface area $\mathrm{SiO}_{2}$ as the disk-shaped $\mathrm{CoO}-\mathrm{NC}$, according to a method described before [49]. The $\mathrm{SiO}_{2}$ had already been pressed and sieved to a fraction of 75-150 $\mu \mathrm{m}$. The support $(500 \mathrm{mg})$ was transferred to a $100 \mathrm{~mL} 3$-necked flask and a mixture of the Co-NC in n-hexane and $6.5 \mathrm{~mL}$ 1-octadecene (90\%, technical grade, Sigma-Aldrich) was 
added under $400 \mathrm{rpm}$ magnetic stirring. The flask was then equipped with a condenser, glass plug and septum and connected to a Schlenk line. The suspension was degassed under vacuum at $100{ }^{\circ} \mathrm{C}$ for $15 \mathrm{~min}$ and as a result, the $\mathrm{n}$-hexane in the suspension evaporated. Then, the flask was flushed with $\mathrm{N}_{2}$ and the deposition was continued under $\mathrm{N}_{2}$. The suspension was heated to $200{ }^{\circ} \mathrm{C}$ for $30 \mathrm{~min}$, after which the heating mantle was removed, causing the solution to cool to room temperature within $30 \mathrm{~min}$. The sample was washed by first centrifuging at $1500 \mathrm{G}$ for $5 \mathrm{~min}$ and decanting the supernatant. Subsequently, the precipitate was resuspended in $2 \mathrm{~mL}$ $\mathrm{n}$-hexane through sonication, $6 \mathrm{~mL}$ acetone was added and the sample was isolated by centrifuging at $1500 \mathrm{G}$ for $5 \mathrm{~min}$ and decanting the supernatant. This precipitation-resuspension cycle was repeated five times, followed by a last cycle in which the $n$-hexane/acetone mixture was replaced by $20 \mathrm{~mL}$ acetone. Finally, the sample was dried in three consecutive steps, i.e. $60{ }^{\circ} \mathrm{C}$ for $1 \mathrm{~h}$ in static air, $120^{\circ} \mathrm{C}$ for $3 \mathrm{~h}$ in static air and $80{ }^{\circ} \mathrm{C}$ for $3 \mathrm{~h}$ under vacuum, and pressed and sieved to a grain size of $75-150 \mu \mathrm{m}$.

\subsection{Characterization}

Transmission electron microscopy (TEM) was performed on a Tecnai 20 (FEI) operated at $200 \mathrm{kV}$. Samples of colloidal suspension were prepared by drop casting a diluted sample directly on a carbon-coated TEM grid. In the case of $\mathrm{SiO}_{2}$-supported $\mathrm{CoO}-\mathrm{NC}$, the powder was first suspended in 2-propanol by sonication and subsequently drop casted on a carbon-coated TEM grid. The TEM images of supported $\mathrm{CoO}-\mathrm{NC}$ were manually analyzed using ImageJ. In the case of the colloidal suspensions, the images of CoO-NC were automatically classified as either disks or spheres and manually reviewed before analysis. All reported NC sizes are number averaged and recalculated to the equivalent $\mathrm{Co}^{0}$ size to compensate for lattice expansion due to a $3 \mathrm{~nm}$ thick $\mathrm{CoO}$ passivation layer. Because of the limited height of the disks, these $\mathrm{NC}$ were assumed to oxidize completely to $\mathrm{CoO}$. The metallic surface area was calculated using the surface area weighted mean diameter, again after correcting for the $3 \mathrm{~nm} \mathrm{CoO}$ passivation layer. A cross-sectional area of 0.0662 $\mathrm{nm}^{2}$ per cobalt atom was assumed in order to calculate the metallic surface area [50].

Vitrification of freshly synthesized colloidal suspensions for cryogenic transmission electron microscopy (cryo-TEM) was performed using a Vitrobot Mark IV (FEI) vitrification robot. The Co-NC were vitrified in the original reaction suspension within $30 \mathrm{~min}$ from magnetically separating the synthesis product. For the vitrification, $3 \mu \mathrm{L}$ of Co-NC suspension was applied to a 200 mesh, lacey carbon-coated $\mathrm{Cu}$ grid at $25{ }^{\circ} \mathrm{C}$ in a chamber saturated with toluene vapor. Subsequently, the grid was blotted for $3 \mathrm{~s}$ with filter paper and plunge-frozen in liquid $\mathrm{N}_{2}\left(-196{ }^{\circ} \mathrm{C}\right)$. The samples were kept in liquid $\mathrm{N}_{2}$ for several hours and loaded into the Titan Krios (FEI) equipped with a field emission gun operating at $300 \mathrm{kV}$ and energy filter. Images were recorded using a $2 \mathrm{k} \times 2 \mathrm{k}$ Gatan charge coupled device (CCD) camera. Highresolution transmission electron microscopy (HR-TEM) was performed in areas where no solvent appeared present. Low dose selective area diffraction (SAED) was used for structure analysis and the diffraction patterns were processed according to the method described by Leijten et al. [51].

Electron tomography was carried out using an FEI Talos F200X operated at $200 \mathrm{kV}$ in bright field TEM mode. The CoO-NC/SiO ${ }_{2}$ sample was suspended in ethanol by ultrasonication. A few droplets of the suspension were deposited onto a Quantifoil R2/1 carbon film supported parallel-bar $\mathrm{Cu}$ grid which already contained Au nanoparticles of $5 \mathrm{~nm}$ in diameter. TEM images were recorded with a Ceta camera $\left(4096 \times 4096\right.$ pixels) over a tilt range of $-6^{\circ}$ to $+76^{\circ}$ with tilt increments of $2^{\circ}$ at a nominal magnification of 74,000 times. The tilt series was aligned using IMOD software [52] using the Au nanoparticles as fiducial markers. The aligned tilt series were binned by 4 and reconstructed using the backprojection algorithm. The resulting reconstructed volumes had a final voxel size of $(0.572 \mathrm{~nm})^{3}$. Segmentation of diskshaped $\mathrm{CoO}-\mathrm{NC}$, spherical $\mathrm{CoO}-\mathrm{NC}$ and the $\mathrm{SiO}_{2}$ support in the reconstructed volumes was performed in FIJI [53]. Adequate threshold values were applied to median-filtered reconstructed volumes. Disk-shaped NC, spherical NC and the $\mathrm{SiO}_{2}$ support were defined in $3 \mathrm{D}$ by manually delimiting their boundary. Volume rendering of the segmented volumes was carried out in FIJI.

Inductively coupled plasma-optical emission spectroscopy (ICP-OES) was performed on a SPECTRO ARCOS after digestion of the sample in aqua regia.

X-ray photoelectron spectroscopy (XPS) was measured on a Kratos Axis Ultra DLD with an X-ray source operating at $225 \mathrm{~W}$ and $15 \mathrm{keV}$, and equipped with an $\mathrm{Al}$ anode $(\mathrm{EK} \alpha=1486.6 \mathrm{eV})$. The samples were fixed using Ag-tape at ambient conditions and loaded in the setup. High-resolution spectra of Co2p, Si2p, O1s and C1s were measured at $40 \mathrm{eV}$ pass energy, while N1s and the survey scan were recorded at $160 \mathrm{eV}$ pass energy. Kratos Axis sensitivity factors were used for quantification. XPS measurements were performed on three distinct locations on all samples and the results were averaged. In case of the N1s scan, multiple measurements of the same location were accumulated to improve the signal-to-noise ratio. The binding energies were calibrated by setting the $\mathrm{C}-\mathrm{C} / \mathrm{C}-\mathrm{H}$ bond peak to $284.8 \mathrm{eV}$.

In situ X-ray diffraction (XRD) was performed on a Bruker D8 Discover with a Mo $\left(\mathrm{K}_{\alpha 1} 0.709 \AA\right)$ source in Debye-Scherrer transmission (capillary) geometry. The $\mathrm{X}$-ray beam was focused on a quartz capillary with $1000 \mu \mathrm{m}$ OD and a wall thickness of $10 \mu \mathrm{m}$ using a Göbel-mirror. The setup was equipped with an energy dispersive LynxEye XE 
Position Sensitive Detector (PSD). Details on the complete setup can be found in recent publications [54, 55]. The patterns were measured between 5 and $39^{\circ} 2 \theta$ with an increment of $0.07^{\circ}$ and time per step of $20.5 \mathrm{~s}$. Multiple scans were accumulated to improve the signal-to-noise ratio only if the patterns were identical. Rietveld Quantitative Phase Analysis (Rietveld QPA) was performed on the measured diffractograms using Bruker TOPAS v5 software. Details of the Rietveld refinement procedure are given in Supporting Methods.

$\mathrm{Co}-\mathrm{S} / \mathrm{SiO}_{2}$ was diluted with 2 mass equivalents $\mathrm{SiO}_{2}$ (Aerosil OX 50) to obtain a similar overall cobalt loading as $\mathrm{Co}-\mathrm{D} / \mathrm{SiO}_{2}$. Typically, $7 \mathrm{mg}$ sample in the sieve fraction $75-150 \mu \mathrm{m}$ was loaded in the capillary and fixed with quartz wool at either end, resulting in a catalyst bed length of $\sim 20 \mathrm{~mm}$. The capillary was placed in the setup and the catalysts were reduced in situ at $350{ }^{\circ} \mathrm{C}$ for $8 \mathrm{~h}\left(1{ }^{\circ} \mathrm{C} \mathrm{min}{ }^{-1}\right)$ in a $1.2 \mathrm{~mL} \mathrm{~min}^{-1}$ flow of $25 \mathrm{vol} \% \mathrm{H}_{2}$ in He at 1 bar. After reduction, the temperature was lowered to $180{ }^{\circ} \mathrm{C}$ with $3{ }^{\circ} \mathrm{C} \mathrm{min}^{-1}$ and at this temperature, a $3.0 \mathrm{~mL} \mathrm{~min}{ }^{-1}$ flow of synthesis gas $\mathrm{H}_{2} / \mathrm{CO}=2(\mathrm{v} / \mathrm{v})$ was introduced. After 10 min stabilization, the back-pressure regulator was set to 10.6 bar. Only after the set pressure was reached and stable, the temperature was increased to $220{ }^{\circ} \mathrm{C}$ with $1{ }^{\circ} \mathrm{C} \mathrm{min}-1$, which was defined as TOS $=0 \mathrm{~h}$. In situ XRD patterns were recorded both during reduction and FT.

\subsection{Fischer-Tropsch Synthesis}

The Fischer-Tropsch synthesis performance of the catalysts was investigated using a Flowrence (Avantium) 16 parallel reactor setup. $80 \mathrm{mg} \mathrm{Co}-\mathrm{D} / \mathrm{SiO}_{2}$ and $12 \mathrm{mg} \mathrm{Co}-\mathrm{S} / \mathrm{SiO}_{2}$ were first diluted with $100 \mathrm{mg} \mathrm{SiC}(212-425 \mu \mathrm{m})$ and then loaded into stainless steel plug-flow reactors of 2.6 and $2.0 \mathrm{~mm}$ (ID), respectively. The samples were dried for $2 \mathrm{~h}$ in an He flow and reduced in situ in a $25 \mathrm{vol} \% \mathrm{H}_{2}$ in He flow at $350{ }^{\circ} \mathrm{C}$ for $8 \mathrm{~h}$ after a heating ramp of $1{ }^{\circ} \mathrm{C} \mathrm{min}{ }^{-1}$. After reduction, the temperature was lowered to $180{ }^{\circ} \mathrm{C}$ with $3{ }^{\circ} \mathrm{C}$ $\min ^{-1}$ and the reactors were pressurized to $20 \mathrm{bar}$ in an $\mathrm{H}_{2}$ flow. Then, the synthesis gas feed was introduced with $\mathrm{H}_{2} /$ $\mathrm{CO}=2 \mathrm{v} / \mathrm{v}$ and $5 \mathrm{vol} \% \mathrm{He}$ as internal standard and after $1 \mathrm{~h}$ stabilization, the reactors were heated to $220{ }^{\circ} \mathrm{C}$ with $1{ }^{\circ} \mathrm{C}$ $\mathrm{min}^{-1}$, which was defined as the starting point for FT. After the run, the catalysts were treated in $\mathrm{H}_{2}$ at $200{ }^{\circ} \mathrm{C}$ for $10 \mathrm{~h}$ to remove carbonaceous products from the catalyst surface and cooled down further under $\mathrm{He}$.

The reaction products were analyzed online using an Agilent 7890A GC with two separate channels. In the first channel, the hydrocarbon products were analyzed using an Agilent J\&W PoraBOND Q column in combination with an FID. In the second channel, the permanent gasses were separated on a ShinCarbon-ST column and analyzed using a TCD. The catalytic activity was given as $\mathrm{CO}$ conversion
$\left(\mathrm{X}_{\mathrm{CO}}\right)$ cobalt-time yield (CTY) and turnover frequency (TOF). The TOF was based on the CTY and the average metallic surface area of the spent catalyst, as determined by TEM. The selectivity (in $\%_{\mathrm{C}}$ ) to $\mathrm{C}_{1}-\mathrm{C}_{4}$ hydrocarbons was determined as $\mathrm{S}_{\mathrm{C} 1-\mathrm{C} 4}=100 \mathrm{~F}_{\mathrm{Cn}} \mathrm{n}\left(\mathrm{F}_{\mathrm{CO}, \text { in }} \mathrm{X}_{\mathrm{CO}}\right)^{-1}$ with $\mathrm{n}$ representing the product's carbon number and $\mathrm{F}$ the flow of the hydrocarbon or $\mathrm{CO}$. The selectivity to $\mathrm{C}_{5+}$ hydrocarbons was calculated as $\mathrm{S}_{\mathrm{C} 5+}=100 \% \mathrm{C}^{-} \mathrm{S}_{\mathrm{C} 1-4}$.

\section{Results and Discussion}

Cobalt nanocrystals (NC) were synthesized by a hot-injection synthesis using oleic acid and octadecylamine as ligands with the aim to synthesize disk-shaped $\mathrm{NC}$ and the results were analyzed by TEM (Fig. 1). Both disk- and sphericalshaped CoO-NC were obtained from a single synthesis with the ratio between the two varying strongly with the reaction conditions, which made separation necessary, in line with literature [10]. Enrichment of the disk-shaped over spherical $\mathrm{NC}$ was realized by a magnetic field, using the difference in the magnetic properties between the different NC. In our case, the large magnetic moment of the spheres resulted in faster precipitation from the solution compared to the disks under the weak magnetic field of the stirring plate (Fig. S1). This led to enhanced concentrations of disks in the top layer of the reaction mixture (Fig. 1a-e). After magnetic separation, the work-up procedure also affected the disk-to-sphere ratio, as the disk yield decreased with more precipitationredispersion cycles (Fig. 1a-c). This indicates that spheres were preferentially precipitated during centrifugation or alternatively, that the fraction of disks already varied in the original reaction mixture of different batches.

The elongated, rod-like particles (mainly visible in Fig. 1a) were identified as stacks of disk-shaped particles orientated side to side. From these particles, the average dimensions of the disks were determined (Fig. S2). The average diameter of the disks was $15.5 \pm 3.5 \mathrm{~nm}$ and the thickness $4.2 \pm 0.6 \mathrm{~nm}$, leading to an aspect ratio (height/ diameter) of 0.27. As a comparison, all-spherical CoO-NC of $8.3 \pm 1.1 \mathrm{~nm}$ were synthesized using only oleic acid as ligand, as described before [49] (Fig. 1f).

A batch containing disk-shaped $\mathrm{NC}$ after three precipitation-redispersion cycles was analyzed in more detail (Fig. 1c). The NC in the TEM image were semi-automatically divided into two groups (Fig. 1e) based on their contrast, which is a measure for their thickness, in relation to their size and morphology. The large, low-contrast NC were (truncated) hexagonally-shaped and the smaller, high-contrast $\mathrm{NC}$ were spherical. To examine the 3D shape of these $\mathrm{NC}$, the stage was tilted $50^{\circ}$ inside the TEM (Fig. 1d, S3 and supporting movie S1). Analysis of $100 \mathrm{NC}$ from both groups (Fig. S4) revealed that the size of the particles was $15.4 \mathrm{~nm}$ 
Fig. 1 TEM images of several batches of mixed spheres/ disks $\mathrm{CoO}-\mathrm{NC}$ after different washing procedures followed by dispersion and drying on the carbon foil (a-e). The washing procedures consisted of a one precipitation-redispersion cycle, b two precipitationredispersion cycles and $\mathbf{c}-\mathbf{e}$ three precipitation-redispersion cycles. c-e Images of the same location with $\mathbf{d}$ showing the sample after tilting by $50^{\circ}$ and $\mathbf{e}$ with an overlay highlighting the particles that were identified as disks (red) and spheres (aqua). f Spherical CoO-NC synthesized using only oleic acid as ligand
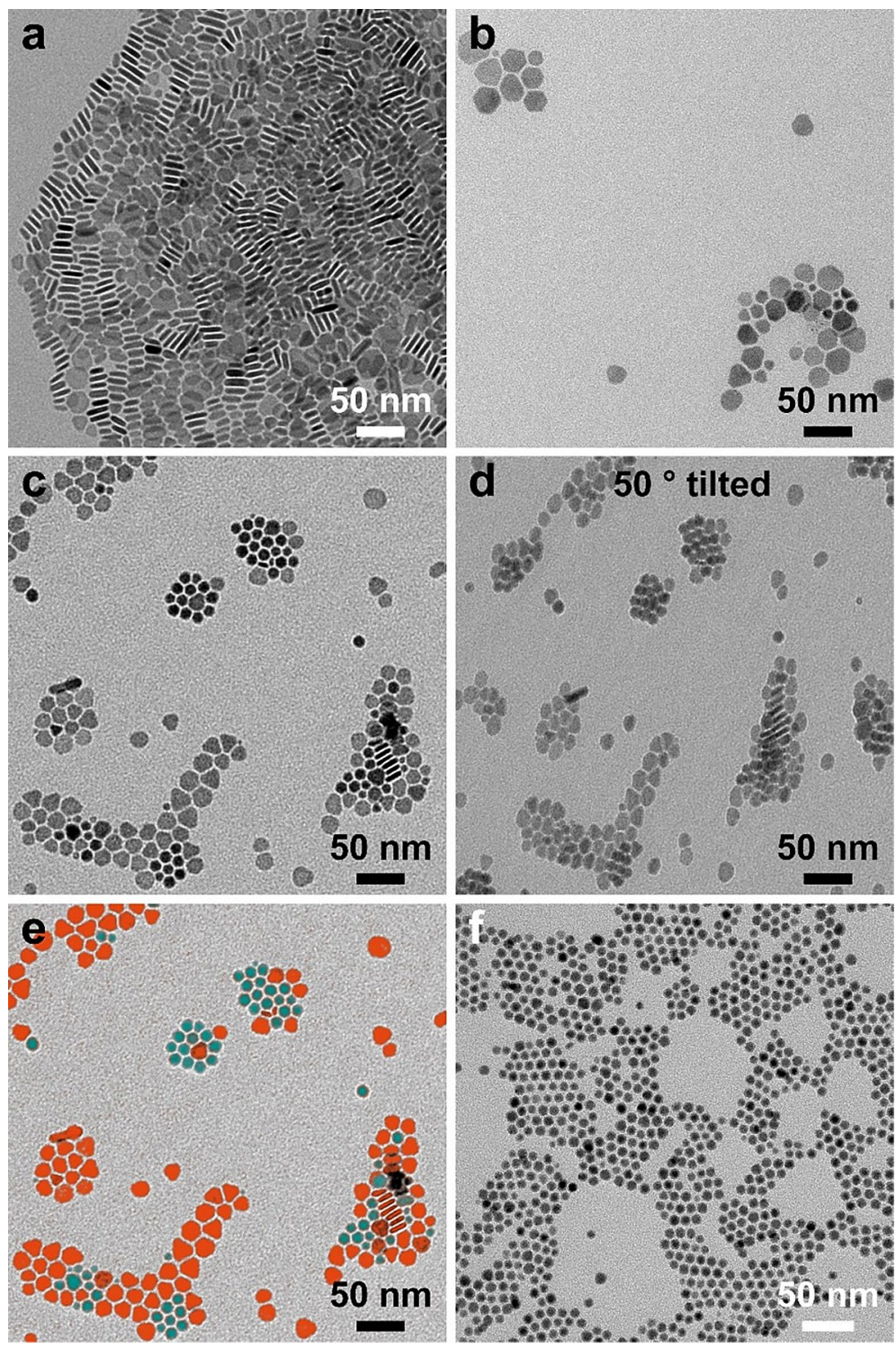

at $0^{\circ}$ and $11.9 \mathrm{~nm}$ at $50^{\circ}$ in the case of the low-contrast particles, proving that these $\mathrm{NC}$ were indeed smaller in the $z$ direction as expected for disk-shaped NC. When considering an aspect ratio of 0.27 , the $2 \mathrm{D}$ projection length at $50^{\circ}$ should be $13.1 \mathrm{~nm}$ (Fig. S5), which matches the experimental value quite well. On the other hand, the smaller, high-contrast particles were $10.7 \mathrm{~nm}$ at $0^{\circ}$ and $10.6 \mathrm{~nm}$ at $50^{\circ}$, showing that their diameter was the same in all directions and that these $\mathrm{NC}$ were indeed spherical. Because the shapes of the CoO-NC could be accurately distinguished, the numerical disk yield could be determined and was $53 \%$ for the batch after three precipitation-redispersion cycles.

Disk-shaped Co-NC are a metastable phase [8, 56], making the synthesis results highly susceptible to small variations in the procedure. Therefore, optimizing the reaction conditions was essential to obtain a high fraction of anisotropic particles. Important synthesis parameters included the speed of magnetic stirring and the speed of precursor injection, as faster stirring or slower injection through a thinner needle led to enhanced formation of 10-30 nm spherical NC and a lower yield of disk-shaped NC. Furthermore, higher disk yields were obtained with batches of $\mathrm{Co}_{2}(\mathrm{CO})_{8}$ that had been aged $40 \mathrm{~min}$ in the glovebox prior to use in synthesis. These phenomena have not been reported before for this synthesis method and illustrate the delicate nature of this approach.

The crystal structure of the as-synthesized Co-NC before oxidation was analyzed using a combined cryoand high-resolution-TEM approach (Fig. 2). A Co-NC suspension was vitrified shortly after NC synthesis and transported and loaded in liquid $\mathrm{N}_{2}$ to avoid oxidation of the Co-NC. The vitrification protected the sample from oxidation and HR-TEM was possible because there was little to no solvent present in the TEM sample. Electron 

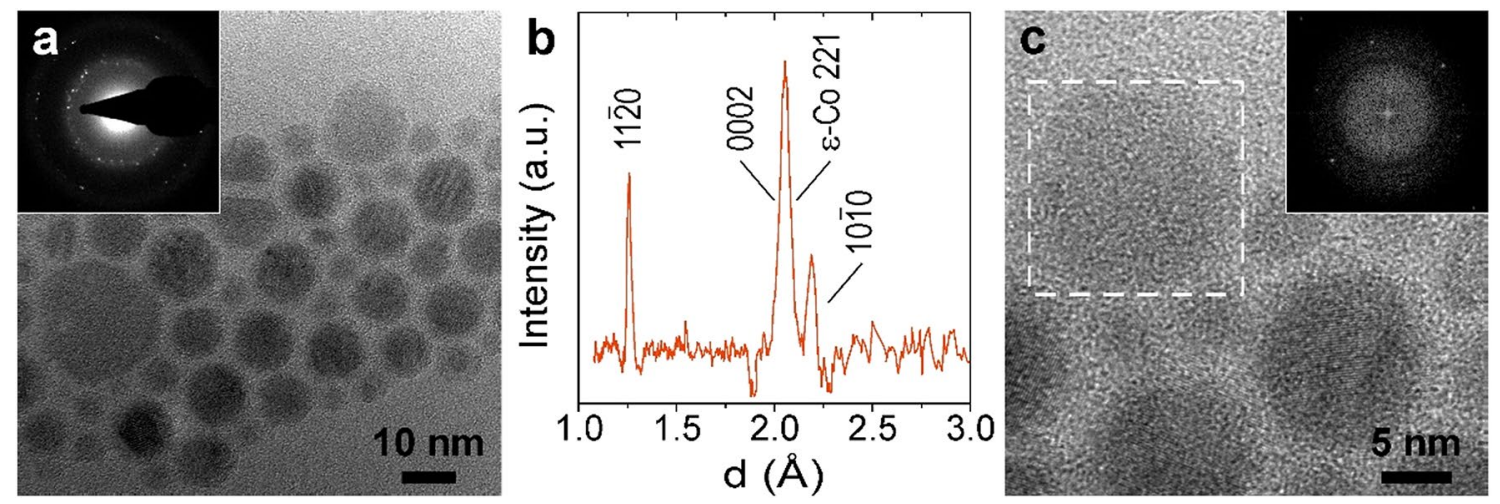

Fig. 2 High-resolution TEM under cryogenic conditions of as-synthesized disk-shaped and spherical Co-NC with little to no solvent present. a Overview of several clustered Co-NC with the selected area electron diffraction pattern in the inset. b Intensity profile

diffraction of the area in Fig. 2a resulted in the diffraction pattern in the inset of Fig. 2a and was radially averaged to give Fig. $2 b$. The main peak at $d=2.06 \AA$ could not be unambiguously assigned to one crystal structure, as this lattice spacing is present in all three metallic cobalt phases (Fig. S6). The diffraction peak at $\mathrm{d}=2.19 \AA$, however, only matched hcp cobalt. Furthermore, the peaks at d-values of 1.26 and $2.19 \AA$ could be ascribed to $\{11 \overline{2} 0\}$ and $\{10 \overline{1} 0\}$ of hcp cobalt, respectively, which were more intense than expected. Considering that $\{11 \overline{2} 0\}$ and $\{10 \overline{1} 0\}$ have periodicity in the $x, y$ direction and not in the $z$ direction, this proved that the hcp cobalt was present in anisotropic, diskshaped NC and that the short $z$ axis is along the [0001] direction, in line with literature [10]. Based on the intensity ratios of the hcp Co phase, it is likely that the peak at $\mathrm{d}=2.06 \AA$ has another dominant contribution either from fcc or $\varepsilon$-cobalt.

Further information on the crystal structure of the NC before oxidation was obtained by investigating an individual $\mathrm{NC}$ in more detail with HR-TEM on the same cryogenic sample. The Co-NC in the framed area in Fig. 2c was identified as a disk-shaped $\mathrm{NC}$ because of its low contrast and diameter of $15 \mathrm{~nm}$. Lattice fringes of this particle were imaged and the Fourier transform revealed hexagonal symmetry of the crystal lattice, indicative of hcp cobalt. The determined lattice spacings were 2.28 and $1.29 \AA$, which were again matched to $\{1 \overline{1} 0\}$ and $\{10 \overline{1} 0\}$ of hcp Co. Additionally, the other prominent particle of $11 \mathrm{~nm}$ with higher contrast was identified as a spherical NC and did not show hexagonal symmetry. Its lattice spacings matched $\varepsilon$-cobalt best (Fig. S7), indicating that the other crystal structure observed by electron diffraction was most likely $\varepsilon$-cobalt, which is consistent with literature [10]. It should be emphasized that this concerned the crystal structure of the Co-NC obtained by radially averaging and baseline correcting the SAED pattern. c Several Co-NC at higher magnification. The framed area contained a $15 \mathrm{~nm}$, low contrast $\mathrm{NC}$ that was classified as a disk; the inset gives the Fourier transform of the framed area

before oxidation and that it is uncertain what crystal structure would evolve after low-temperature oxidation, in situ reduction and catalysis.

The CoO-NC after two precipitation-redispersion cycles were deposited on low surface area $\mathrm{SiO}_{2}\left(50 \mathrm{~m}^{2} \mathrm{~g}^{-1}\right)$ using a method that avoids the use of high temperatures to affect the NC shape as little as possible. This particular type of $\mathrm{SiO}_{2}$ was selected, because it had yielded very stable Co-NC based catalysts under FT conditions before [49]. The sample containing mainly disk-shaped $\mathrm{NC}$ was designated as Co-D/ $\mathrm{SiO}_{2}$. Two washing steps after $\mathrm{NC}$ synthesis were the optimal compromise between uniform attachment of the $\mathrm{CoO}-$ $\mathrm{NC}$ with lower ligand concentration on one hand (Fig. S8) and progressively lower NC yields on the other. Based on ICP-OES, a cobalt loading of $1.4 \mathrm{wt} \%$ was obtained using this deposition method. The $\mathrm{Co}-\mathrm{D} / \mathrm{SiO}_{2}$ sample was analyzed by TEM (Fig. 3a-c). The NC were largely uniformly distributed over the support, although some areas with clustered disk-shaped and spherical $\mathrm{NC}$ were encountered as well (Fig. 3c). Despite this occasional clustering of NC, NC were largely well dispersed on the support, making the catalyst useful for further investigations.

It was challenging to distinguish the disk-shaped $\mathrm{NC}$ from the $\mathrm{SiO}_{2}$ support because of the low contrast of the CoO$\mathrm{NC}$ associated with their limited thickness when orientated perpendicular to the electron beam. However, based on a combination of contrast, size and morphology, it was possible to identify the $\mathrm{CoO}-\mathrm{NC}$ and discriminate between disks and spheres (Fig. 3a, b). After analysis of $203 \mathrm{NC}, 66 \%$ of the $\mathrm{NC}$ were classified as disks and $34 \%$ as spheres. In addition, a reference catalyst containing exclusively spherical $\mathrm{CoO}-\mathrm{NC}$ was prepared by depositing the $8.3 \mathrm{~nm} \mathrm{CoO}-\mathrm{NC}$ on the same low surface area $\mathrm{SiO}_{2}$ (Fig. 3d). This catalyst was designated as $\mathrm{Co}-\mathrm{S} / \mathrm{SiO}_{2}$. The obtained cobalt loading was $9.6 \mathrm{wt} \%$ based on ICP-OES. Furthermore, the $\mathrm{CoO}-\mathrm{NC}$ were 
Fig. 3 TEM images of pristine samples of CoO-NC deposited on $\mathrm{SiO}_{2}$. a An area of Co-D/ $\mathrm{SiO}_{2}$ with an uniform distribution of CoO-NC and $\mathbf{b}$ the same location with an overlay highlighting the $\mathrm{NC}$ that were identified as disks (red) and spheres (aqua). c An area of Co-D/ $\mathrm{SiO}_{2}$ where the $\mathrm{CoO}-\mathrm{NC}$ were clustered. d Co-S/SiO with an uniform $\mathrm{CoO}-\mathrm{NC}$ distribution

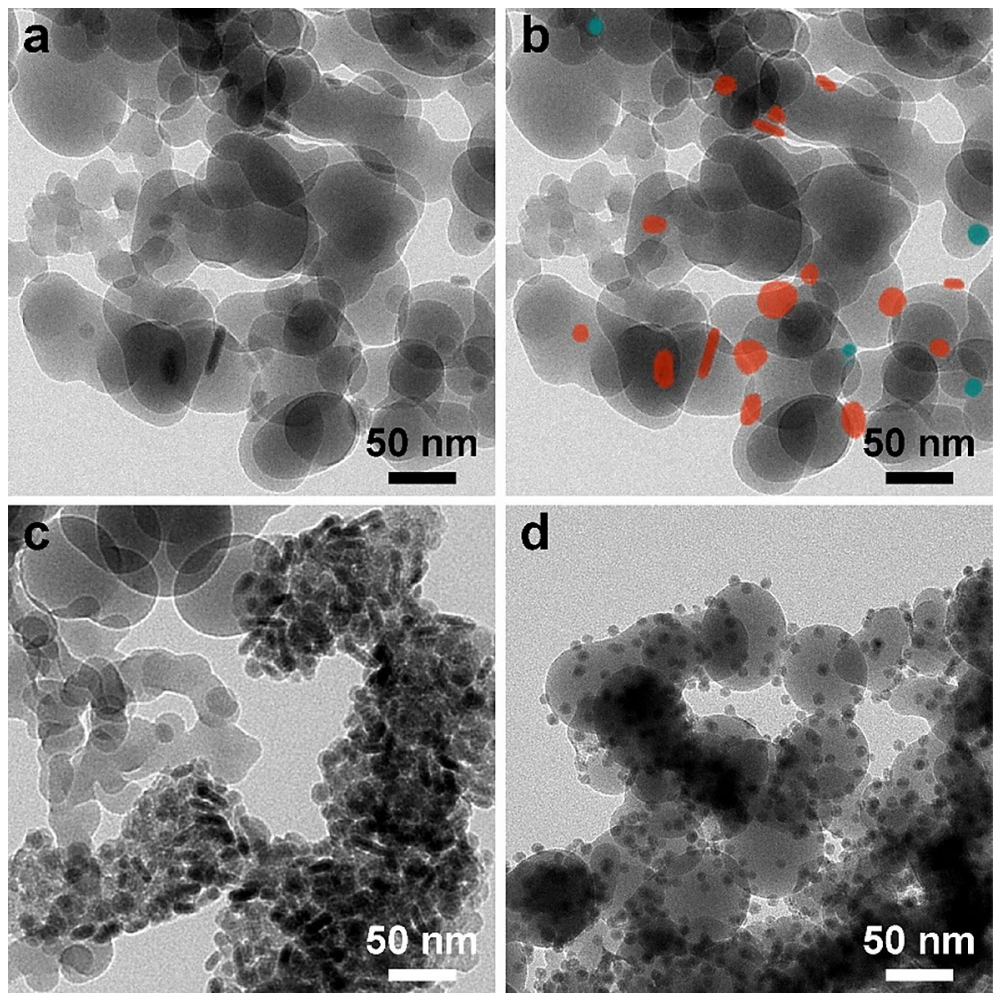

uniformly distributed over the support and no areas containing clustered $\mathrm{NC}$ were observed. This catalyst was described in more detail in an earlier publication [49].

Electron tomography of $\mathrm{Co}-\mathrm{D} / \mathrm{SiO}_{2}$ was performed to investigate the morphology of the $\mathrm{NC}$ on the support in more detail (Fig. 4, complete tilt series from -66 to $+76^{\circ}$ included as supporting movie $\mathrm{S} 2$ ). The disks could be clearly distinguished from the spheres using the tilt series, because only the projected aspect ratio of the disks varied with the tilting angle. The accuracy of the method applied before to classify the NC as disks or spheres based on size, morphology and contrast was confirmed by tomography and the obtained results for this limited sample size (only $26 \mathrm{NC}$ in field of view) matched the earlier observation of $~ 65 \%$ of the NC present as disks. Furthermore, a 3D representation of a selected part of the sample was obtained through reconstruction and segmentation of the tilt series (Fig. 4c, reconstruction and 3D-rendered volume as supporting movies S3, S4). From this representation, the morphology of NC can be clearly inferred as well as their interaction with the support. It showed that the disk-shaped $\mathrm{NC}$ typically attached to $\mathrm{SiO}_{2}$ with one base plane. Future work on metal-support interactions for the differently shaped NC could involve, e.g., XPS experiments to explore electronic interactions of the Co-NC with the support.

The FT performance of the catalysts was evaluated over $100 \mathrm{~h}$ on stream at similar CO conversion levels (Fig. 5, Table 1). The cobalt-weight-based activity (cobalt-time yield, CTY) against TOS is shown in Fig. 5b. Co-S/SiO was twice as active per unit weight of cobalt as $\mathrm{Co}-\mathrm{D} / \mathrm{SiO}_{2}$. Because of the different particle sizes and shapes, the activity was compared as cobalt-surface-based activity (turnover frequency, TOF). The metallic surface area necessary for the TOF was calculated from the NC sizes in the spent catalyst, which will be discussed later (Table 1). The TOF of Co-D/ $\mathrm{SiO}_{2}$ was $49 \times 10^{-3} \mathrm{~s}^{-1}$ and the TOF of $\mathrm{Co}-\mathrm{S} / \mathrm{SiO}_{2}$ was $86 \times$ $10^{-3} \mathrm{~s}^{-1}$, the former value being in line with other work on $\mathrm{Co} / \mathrm{SiO}_{2}$ using conventional catalyst preparation techniques such as impregnation [57]. However, the TOF of $\mathrm{Co}-\mathrm{S} / \mathrm{SiO}_{2}$ was remarkably high, possibly pointing to a higher fraction of more active hcp Co [22-26] resulting from the NC-based synthesis compared to conventional synthesis methods.

The selectivities towards $\mathrm{C}_{5+}$, as well as $\mathrm{C}_{1}$ - and $\mathrm{C}_{2}-\mathrm{C}_{4}$, products were similar (Table 1 ) and overall $\sim 5 \%$ lower than on conventionally prepared $\mathrm{Co} / \mathrm{SiO}_{2}$ catalysts [57]. Consequently, the ASF distributions and derived chain growth probabilities $(\alpha)$ were comparable as well. Plots of the $\mathrm{C}_{5+}$-selectivity as a function of TOS and the ASF distributions are included as Fig. S9. The effect of crystal structure on selectivity is still under debate, as some reports indicated no difference between hcp and fcc Co [22, 27], while others observed higher $\mathrm{C}_{5+}$-selectivity on hcp Co [24, 58]. However, in our case, similar $\mathrm{C}_{5+}$-selectivity of both catalysts is in line with expectations when considering that hcp (0001) surfaces are virtually inactive compared to higher index surfaces. Therefore, if (0001) concentrations for Co-D/ 

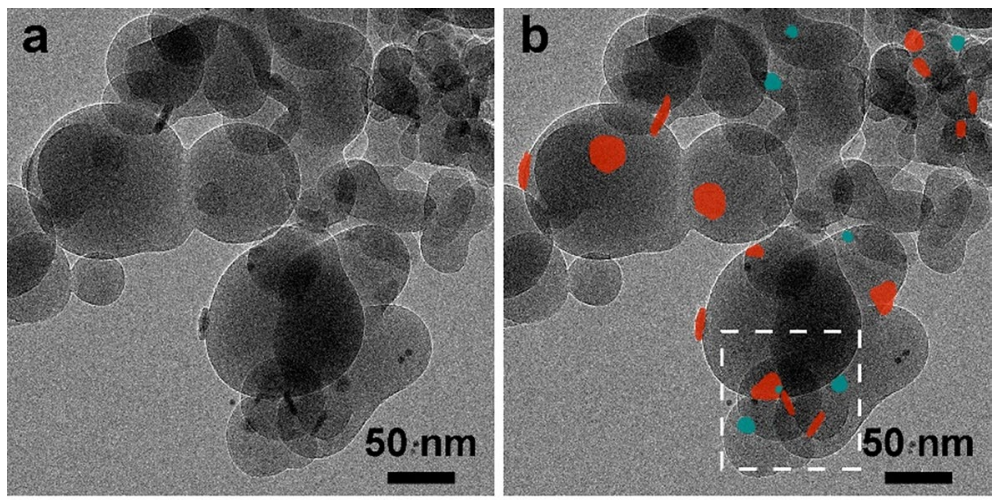

C
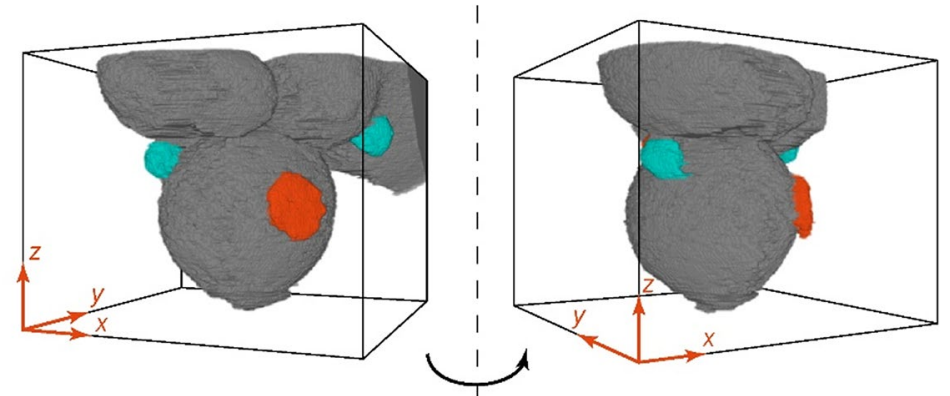

Fig. 4 Electron tomography results of the pristine $\mathrm{Co}-\mathrm{D} / \mathrm{SiO}_{2}$ sample, consisting of a mixture of disk-shaped and spherical CoO-NC deposited on $\mathrm{SiO}_{2}$. a TEM image of $\mathrm{Co}-\mathrm{D} / \mathrm{SiO}_{2}$ at $0^{\circ}$ tilt angle. The small $(\sim 5 \mathrm{~nm})$, high-contrast particles are gold fudicial markers on the TEM grid. b The same TEM image with an overlay highlighting the NC that were identified as disks (red) and spheres (aqua). This location was reconstructed and the framed area was segmented. c Volume-rendered 3D reconstruction of the framed area in $\mathbf{b}$, shown

$\mathrm{SiO}_{2}$ were to be enhanced, these additional surfaces would contribute only marginally to the overall product spectrum and as a result, the selectivity would hardly change. Thus, changing the $\mathrm{NC}$ shape from spheres to disks did not influence the selectivity of the catalyst.

Overall, the main difference in performance was the factor 2 lower intrinsic activity of the disk-containing Co-D/ $\mathrm{SiO}_{2}$ sample, which could have several reasons. First, the activity could be lower as a result of the shape and thus the exposed facets, which would match the theoretical and experimental finding that hop (0001) is less active than higher-index hcp or fcc facets [25, 32]. Second, residual ligands could still be present in the case of $\mathrm{Co}-\mathrm{D} / \mathrm{SiO}_{2}$ and block part of the cobalt surface. Third, the surface area of Co-D/SiO 2 could be lower than expected if the degree of reduction is low or if there is substantial $\mathrm{NC}$ growth, for example in the areas where clustering of $\mathrm{NC}$ was observed (Fig. 3c). These potential explanations were investigated in more detail and are described below.

The structure of the spent catalysts was analyzed after FT and subsequent passivation using TEM (Fig. 6). The at two different angles obtained by rotation along the $z$ axis (dashed line). The $z$ axis also indicates the direction of the projection in the original TEM image a, b. The $\mathrm{SiO}_{2}$ support is depicted in grey, diskshaped $\mathrm{CoO}-\mathrm{NC}$ in red and spherical $\mathrm{CoO}-\mathrm{NC}$ in aqua. An animation showing a $360^{\circ}$ rotation of this segmented sample together with the tilt series and the full reconstruction file are included as supporting movies S2-4

sizes and shapes of 160 cobalt nanocrystals in $\mathrm{Co}-\mathrm{D} / \mathrm{SiO}_{2}$ were quantified (Fig. 6a, b), showing numerically $62 \%$ disk-shaped $\mathrm{NC}$ with $\mathrm{d}=14.5 \mathrm{~nm}$ and $38 \%$ spherical $\mathrm{NC}$ with $\mathrm{d}=10.2 \mathrm{~nm}$. The aspect ratio of the disks could not be reliably determined for this sample and was assumed to have remained unchanged. These results were very similar to those of the pristine catalyst and the disk shape was thus remarkably stable under FT conditions. Furthermore, a more clustered part of the catalyst was also observed where some particle growth had occurred (Fig. 6c). This area probably originated from an area of clustered $\mathrm{CoO}-\mathrm{NC}$ in the pristine catalyst. The size of the $\mathrm{NC}$ in $\mathrm{Co}-\mathrm{S} / \mathrm{SiO}_{2}$ was unchanged as well with spherical particles of $8.7 \mathrm{~nm}$ on average (Fig. 6d). The stability of the Co-NC in terms of both size and shape under reaction conditions meant that catalytic performance might be related to the disk shape.

Two types of ligands were used to synthesize diskshaped Co-NC (oleic acid and octadecylamine) while spherical Co-NC were synthesized using only oleic acid. For oleic acid, efficient ligand removal has been shown [7], but could be different for linear amines. We assessed the 

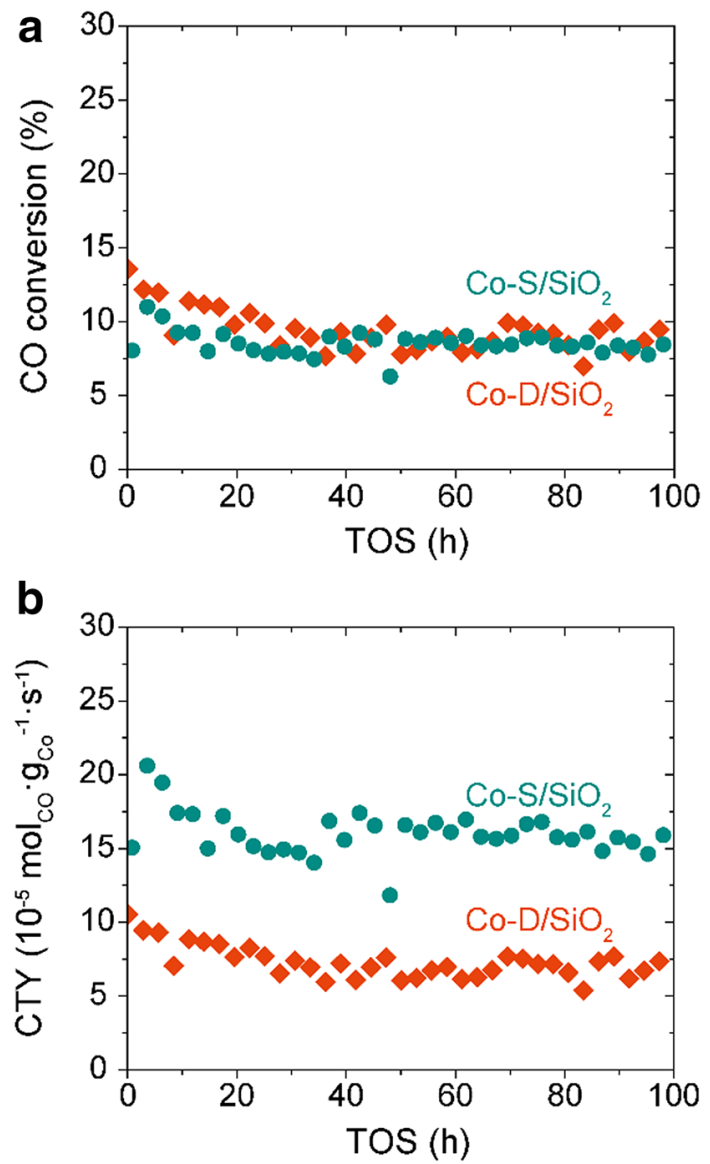

Fig. 5 Fischer-Tropsch synthesis results of $\mathrm{Co}-\mathrm{D} / \mathrm{SiO}_{2}$ and $\mathrm{Co}-\mathrm{S} /$ $\mathrm{SiO}_{2}$ at $20 \mathrm{bar}, 220{ }^{\circ} \mathrm{C}$ and $\mathrm{H}_{2} / \mathrm{CO}=2 \mathrm{v} / \mathrm{v}$. The GHSV was $1560 \mathrm{~h}^{-1}$ for $\mathrm{Co}-\mathrm{D} / \mathrm{SiO}_{2}$ and $39,900 \mathrm{~h}^{-1}$ for $\mathrm{Co}-\mathrm{S} / \mathrm{SiO}_{2}$. a CO conversion and $\mathbf{b}$ cobalt-time yield as a function of time-on-stream

influence of the octadecylamine ligand in FT by investigating the amount of surface nitrogen species using XPS on the catalysts as-prepared and after $100 \mathrm{~h}$ FT followed by passivation. Low concentrations of $\mathrm{N}$ were detected on both as-prepared catalysts and on bare $\mathrm{SiO}_{2}$ (Fig. 7a, complete survey scans in Fig. S10) The N-to-Co atom ratio was 4 times higher on $\mathrm{Co}-\mathrm{D} / \mathrm{SiO}_{2}(0.07)$ than on $\mathrm{Co}-\mathrm{S} /$ $\mathrm{SiO}_{2}(0.02)$. After FT and passivation, $\mathrm{Co}-\mathrm{D} / \mathrm{SiO}_{2}$ was partially covered in carbon and only an indication of $\mathrm{N}$ was observed. Spent $\mathrm{Co}-\mathrm{S} / \mathrm{SiO}_{2}$ was too covered in carbon to yield useful data on Co and $\mathrm{N}$ contents. The N/Co atom ratio of spent $\mathrm{Co}-\mathrm{D} / \mathrm{SiO}_{2}$ seemed similar to that of as-prepared $\mathrm{Co}-\mathrm{S} / \mathrm{SiO}_{2}$, so excess $\mathrm{N}$-species in Co-D/SiO from the $\mathrm{NC}$ synthesis procedure were removed during reduction/FT. Harmel et al. [46] quantified that the content of the ligand hexadecylamine decreased from $8 \mathrm{wt} \%$ to $3 \mathrm{wt} \%$ during reduction under $\mathrm{H}_{2}$ at $270{ }^{\circ} \mathrm{C}$, which is substantially lower than the reduction temperature of $350^{\circ} \mathrm{C}$ applied here. Therefore, it seemed that our reduction procedure was sufficient to activate the catalyst and minimize the effect of remaining $\mathrm{N}$-species.

To get more insight into the structure of the catalysts under FT conditions, in situ XRD was performed on both catalysts at 10 bar, $220{ }^{\circ} \mathrm{C}$ and an $\mathrm{H}_{2} / \mathrm{CO}$ ratio of $2 \mathrm{v} / \mathrm{v}$ (Fig. 7b, Fig. S11). To obtain satisfying Rietveld refinement results, it was necessary to allow preferred orientations of hcp Co crystallites, similar to what has been reported before [55]. We ascribe this to polymorphism of Co in the Co-NC/SiO ${ }_{2}$ samples, since XRD analysis of metallic cobalt is complicated by the propensity of cobalt to include stacking faults $[22,59]$. Our analysis method did not quantify this intergrowth structure, but accommodated much of it in the hcp contribution by increasing the intensity of the (0002) peak. The fraction of hcp Co could therefore be overestimated. However, both samples were refined using the same method and given the similarity of the results for both samples, it appears that polymorphism, if present, took place to the same extent in both samples.

The crystalline cobalt phase in both catalysts consisted of approximately $65 \% \mathrm{hcp} \mathrm{Co}$. The fcc $\mathrm{Co}$ and $\mathrm{CoO}$ compositions were $21 \% \mathrm{fcc} \mathrm{Co}$ and $12 \% \mathrm{CoO}$ for $\mathrm{Co}-\mathrm{D} / \mathrm{SiO}_{2}$ and $32 \%$ fcc $\mathrm{Co}$ and $3 \% \mathrm{CoO}$ for $\mathrm{Co}-\mathrm{S} / \mathrm{SiO}_{2}$. These values are in line with results obtained by Andreev et al. [60] using $\mathrm{NMR}$ on $\mathrm{Co} / \mathrm{SiC}$ at elevated temperatures under static Ar atmosphere and in reasonable agreement with Cats et al. [54] who used operando XRD on $\mathrm{Co} / \mathrm{TiO}_{2}$ under FT conditions. In general, however, direct comparison of these results to literature is cumbersome, since the obtained Co phase strongly depends on particle size, metal precursor, support and activation procedure [61, 62]. Furthermore, the average crystallite sizes of metallic cobalt

Table 1 Fischer-Tropsch synthesis results for $\mathrm{Co}-\mathrm{D} / \mathrm{SiO}_{2}$ and $\mathrm{Co}-\mathrm{S} / \mathrm{SiO}_{2}$ reported at similar $\mathrm{CO}$ conversions and averaged between 90 and $100 \mathrm{~h}$ on stream at $20 \mathrm{bar}, 220^{\circ} \mathrm{C}$ and $\mathrm{H}_{2} / \mathrm{CO}=2 \mathrm{v} / \mathrm{v}$

\begin{tabular}{|c|c|c|c|c|c|c|c|c|c|}
\hline Sample & GHSV $\left(h^{-1}\right)$ & $\mathrm{X}_{\mathrm{CO}}(\%)$ & $\mathrm{CTY}^{\mathrm{a}}$ & $\begin{array}{l}\text { TOF }_{\text {app }} \\
\left(10^{-3} \mathrm{~s}^{-1}\right)^{\mathrm{b}}\end{array}$ & $\mathrm{C}_{5+}$-sel. $\left(\%_{\mathrm{C}}\right)$ & $\begin{array}{l}\mathrm{C}_{2}-\mathrm{C}_{4} \text {-sel. } \\
\left(\%_{\mathrm{C}}\right)\end{array}$ & $\mathrm{C}_{1}$-sel. $\left(\%_{\mathrm{C}}\right)$ & $\alpha$ & $\begin{array}{l}\text { Co SA spent } \\
\text { catalysts }\left(\mathrm{m}^{2}\right. \\
\left.\mathrm{g}_{\mathrm{Co}}{ }^{-1}\right)\end{array}$ \\
\hline $\mathrm{Co}-\mathrm{D} / \mathrm{SiO}_{2}$ & 1560 & 8.9 & 6.9 & 49 & 75 & 8.6 & 16 & 0.78 & 56 \\
\hline $\mathrm{Co}-\mathrm{S} / \mathrm{SiO}_{2}$ & 39,900 & 8.1 & 15 & 86 & 78 & 9 & 13 & 0.76 & 71 \\
\hline
\end{tabular}

${ }^{\mathrm{a}}$ In $10^{-5} \mathrm{~mol}_{\mathrm{CO}} \mathrm{g}_{\mathrm{Co}}{ }^{-1} \mathrm{~s}^{-1}$

${ }^{\mathrm{b}}$ Based on the CTY and the metallic Co surface area of the spent catalysts as derived by TEM 
Fig. 6 TEM images of $\mathrm{Co} /$ $\mathrm{SiO}_{2}$ samples after $>100 \mathrm{~h}$ of Fischer-Tropsch synthesis and passivation. a An area of Co-D/ $\mathrm{SiO}_{2}$ with an uniform distribution of NC and $\mathbf{b}$ the same location with an overlay highlighting the $\mathrm{NC}$ that were identified as disks (red) and spheres (aqua). c An area of $\mathrm{Co}-\mathrm{D} / \mathrm{SiO}_{2}$ where the $\mathrm{NC}$ were clustered. d $\mathrm{Co}-\mathrm{S} / \mathrm{SiO}_{2}$ with a homogeneous $\mathrm{NC}$ distribution
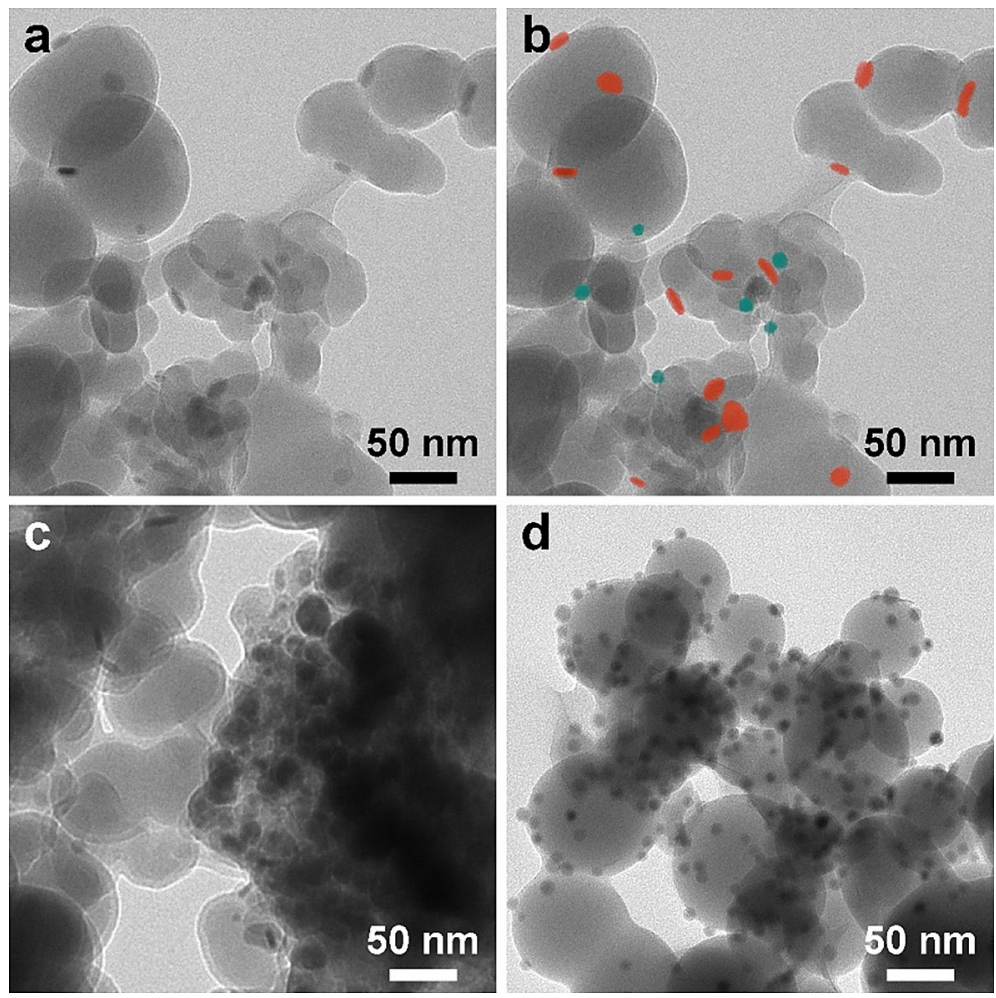

was 3-5 nm, significantly smaller than the particle dimensions obtained by TEM. This probably indicated that the Co-NC were polycrystalline, which could be related to the occurrence of stacking faults.

The degree of reduction of the crystalline phase was high with $88 \%$ for $\mathrm{Co}-\mathrm{D} / \mathrm{SiO}_{2}$ and $97 \%$ for $\mathrm{Co}-\mathrm{S} / \mathrm{SiO}_{2}$ and therefore excluded as a reason for substantial activity differences. Once in the reduced state, the XRD profiles remained unchanged for the duration of the experiment. The crystallites thus remained stable without sintering or restructuring during reduction and the first $18 \mathrm{~h} \mathrm{FT}$, in line with the TEM analyses. This also indicated that a significant loss of surface area in $\mathrm{Co}-\mathrm{D} / \mathrm{SiO}_{2}$ due to sintering of $\mathrm{NC}$ was unlikely. Furthermore, the hcp Co content in $\mathrm{Co}-\mathrm{D} / \mathrm{SiO}_{2}$ was similar to that in $\mathrm{Co}-\mathrm{S} / \mathrm{SiO}_{2}$, but nevertheless substantial in both samples. Finally, the average crystallite dimensions of 3-5 nm prevented any conclusions on preferred orientations and surface termination in disk-shaped NC with a diameter of $16 \mathrm{~nm}$ and $4 \mathrm{~nm}$ thickness. Overall, activity differences due to enhanced hcp Co content was excluded, but preferred crystallite orientations within the polycrystalline disk-shaped NC and with that enhanced hcp (0001) terminations were still possible.

Combining the obtained data on the ligands and NC size, shape and crystal structure of the catalysts under reaction conditions, the cobalt specific surface area and the fraction of the surface terminated by hcp (0001) were calculated (Fig. 8). This served as an estimation of the structural difference between the Co-NC disk-containing catalyst and the catalyst containing exclusively spherical NC. To do so, we assumed that the base planes of the disks were terminated by hcp (0001) and that the disks were attached to $\mathrm{SiO}_{2}$ with one base plane (Fig. 4, supporting movie S4), thereby rendering that plane inaccessible for reactants. This is a conservative estimate, as the curvature of the $\mathrm{SiO}_{2}$ might in practice make these base planes partially accessible, which would increase the relative occurrence of hcp (0001). Furthermore, the dimensions of the as-synthesized NC were used in surface area calculations, i.e. disks with $\mathrm{d}=15.5 \mathrm{~nm}$ and $\mathrm{h}=4.2 \mathrm{~nm}$ and spheres with $\mathrm{d}=10.7 \mathrm{~nm}$.

Besides the surface of the disks, also the surface of the spheres has to be considered. According to Wulff reconstructions of spherical hcp cobalt particles by Liu et al. [25], 18\% of the surface of a spherical hcp cobalt particle is terminated by (0001). Therefore, the maximal hcp (0001) occurrence in $\mathrm{Co}-\mathrm{S} / \mathrm{SiO}_{2}$ was $18 \%$. Compared to an estimated hcp $(0001)$ occurrence of $39 \%$ in $\mathrm{Co}-\mathrm{D} / \mathrm{SiO}_{2}$, this means that $21 \%$ of the metallic cobalt surface area of $\mathrm{Co}-\mathrm{S} / \mathrm{SiO}_{2}$ is in a higher index and probably more active form than for the disk-containing $\mathrm{Co}-\mathrm{D} / \mathrm{SiO}_{2}$. This holds when all $\mathrm{NC}$ consist of hcp Co, while a difference in hcp (0001) exposure of 33\% would be obtained if only disks were hcp Co. Considering the uncertainty and error margin in this analysis, we conclude that the difference in hcp (0001) exposure could be of the right magnitude to account for much of the observed activity difference. Therefore, we identified this as a plausible cause for 


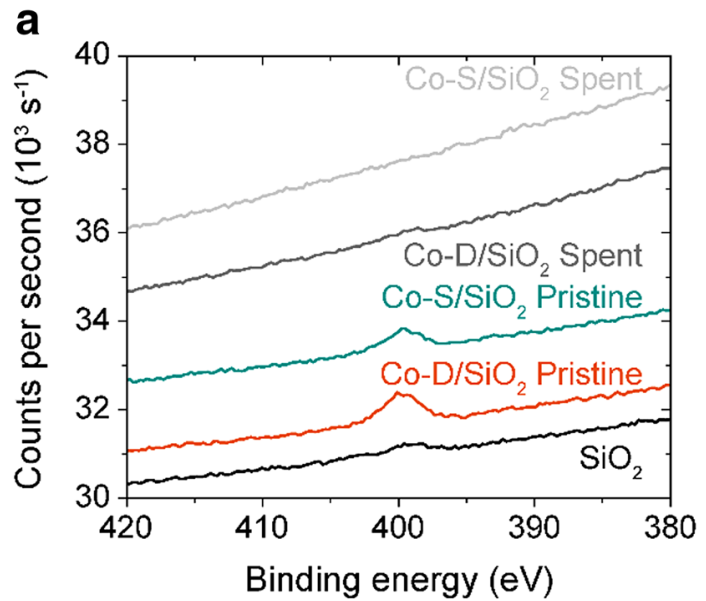

b

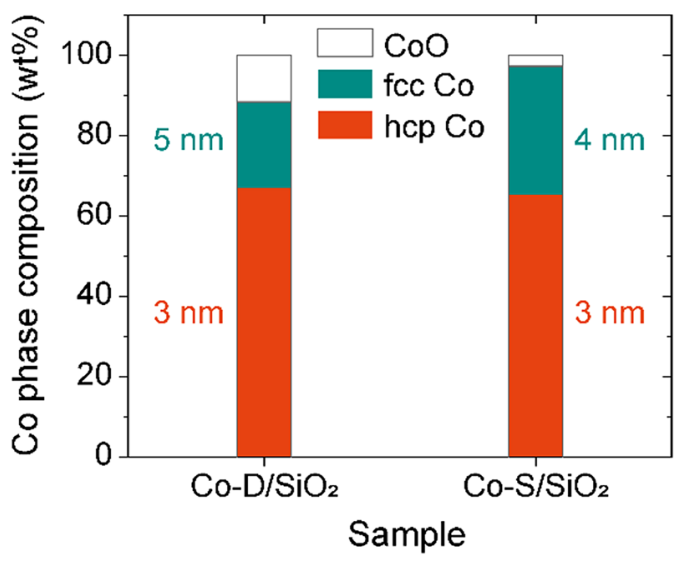

Fig. 7 Nitrogen species on the catalysts before and after $100 \mathrm{~h}$ FT followed by passivation and cobalt crystal structure during FT. a N1s XPS signal around $400 \mathrm{eV}$ of the catalysts in the pristine and spent state and a bare $\mathrm{SiO}_{2}$ reference. The data was offset with 1500 CPS increment at $380 \mathrm{eV}$ binding energy, except for $\mathrm{Co}-\mathrm{S} / \mathrm{SiO}_{2}$ spent (offset -8000 CPS). b cobalt phase composition during Fischer-Tropsch synthesis as determined using in situ XRD and Rietveld refinement. The average crystallite sizes per metallic Co phase are indicated next to the corresponding columns. The amount of $\mathrm{CoO}$ was too low to reliably determine a crystallite size. The diffractograms were obtained over the first $18 \mathrm{~h}$ on stream at $10 \mathrm{bar}, 220^{\circ} \mathrm{C}$ and $\mathrm{H}_{2} / \mathrm{CO}=2 \mathrm{v} / \mathrm{v}$ by accumulating six scans of $3 \mathrm{~h}$. No change in the diffraction patterns was observed over the course of the reaction

the differences in catalytic performance of the disk-shaped and spherical NC, also because of the exclusion of other factors that might lead to such a substantial loss of activity, e.g. surface coverage by ligands, or NC restructuring or growth, as discussed before.

This is, to the best of our knowledge, the first time that a supported model catalyst has been synthesized to investigate the effect of Co-NC shape in FT without complications of a secondary metal or interference of ligands and it illustrates the potential of colloidal techniques for model catalyst synthesis. This work shows that nanoparticle shape can be an important design parameter for supported cobalt FT catalysts

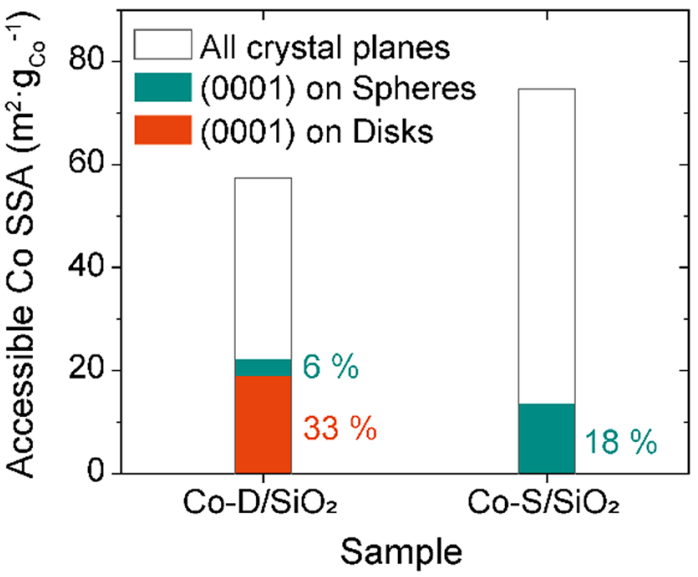

Fig. 8 Calculated accessible Co specific surface area (open bars) of $\mathrm{Co}-\mathrm{D} / \mathrm{SiO}_{2}$ and $\mathrm{Co}-\mathrm{S} / \mathrm{SiO}_{2}$ under FT conditions. The hcp (0001) surface area is indicated and broken down for the disk-shaped (red) and spherical (aqua) Co-NC

when operated under industrially relevant conditions. Furthermore, based on these results, we tentatively propose that the extended low-index surface facets of the disks are the main contributor to the lower TOF.

\section{Conclusions}

We investigated the synthesis of disk-shaped cobalt nanocrystals (Co-NC), their deposition on a support and the subsequent effect of their anisotropy on the Fischer-Tropsch synthesis. Disk-shaped hcp Co-NC were synthesized mixed with spherical $\varepsilon$ Co-NC. After oxidation to $\mathrm{CoO}$, these $\mathrm{NC}$ were deposited on low surface area silica. Electron microscopy analysis revealed that numerically $66 \%$ of the deposited CoO-NC were disks and 34\% were spheres. After in situ reduction of the $\mathrm{CoO}-\mathrm{NC}$ to $\mathrm{Co}$, the Fischer-Tropsch activity of disk-containing catalyst was only half that of a catalyst containing only spherical NC. Interestingly, the catalysts were stable and the size and shape of the NC were retained, even for the disk-shaped Co-NC. XPS showed that most of the amine ligands were removed during activation/FT and in situ XRD revealed that the crystal structure of both catalysts was similar, with $\mathrm{NC}$ with $3-5 \mathrm{~nm}$ polycrystalline domains and approximately $65 \%$ hcp Co and 21 to $32 \%$ fcc Co. Based on these experiments, we tentatively propose that changing the Co-NC shape from spheres to disks reduced FT activity because of hcp (0001) facets prevailing. This work clearly demonstrates the potential of colloidal methods to synthesize new, well-defined model catalysts to investigate structure-performance relationships in catalysis even under high-pressure conditions. 
Acknowledgements T.W.v.D., H.S., B.M.W. and K.P.d.J. acknowledge Shell Global Solutions and the Netherlands Association for Scientific Research (NWO) for funding through the CHIPP framework. H.Y., J.Z. and K.P.d.J. acknowledge the European Research Council, EU FP7 ERC Advanced Grant No. 338846. H.Y. acknowledges the Program for Advancing Strategic International Networks to Accelerate the Circulation of Talented Researchers by JSPS. We thank Nico Sommerdijk, Hans Meeldijk and Paul Bomans for their involvement with the (cryo-) TEM measurements, and Peter Munnik, Peter van den Brink, Sander van Bavel and Marco de Ridder from Shell Technology Center Amsterdam for XPS measurements and useful discussions.

Author Contributions TWD synthesized and characterized the catalysts, evaluated their catalytic performance and drafted the manuscript. $\mathrm{JH}$ assisted with sample preparation and JJN synthesized the reference catalyst. HS performed cryo-TEM and interpretation, HY and JZ were involved with tomography and reconstruction, and RO and BMW performed in situ XRD and assisted with data analysis. KPJ contributed to experimental design, data analysis and writing of the manuscript. All authors have given approval to the final version of the manuscript.

Funding This work was funded by Shell Global Solutions and the Netherlands Association for Scientific Research (NWO) for funding through the CHIPP framework. Further financial support was obtained from the European Research Council, EU FP7 ERC Advanced Grant No. 338846 and the Program for Advancing Strategic International Networks to Accelerate the Circulation of Talented Researchers by JSPS.

Data Availability Data is available upon reasonable request.

\section{Compliance with Ethical Standards}

Conflict of interest There are no conflicts of interest to declare.

Open Access This article is licensed under a Creative Commons Attribution 4.0 International License, which permits use, sharing, adaptation, distribution and reproduction in any medium or format, as long as you give appropriate credit to the original author(s) and the source, provide a link to the Creative Commons licence, and indicate if changes were made. The images or other third party material in this article are included in the article's Creative Commons licence, unless indicated otherwise in a credit line to the material. If material is not included in the article's Creative Commons licence and your intended use is not permitted by statutory regulation or exceeds the permitted use, you will need to obtain permission directly from the copyright holder. To view a copy of this licence, visit http://creativecommons.org/licenses/by/4.0/.

\section{References}

1. Zečević J, Vanbutsele G, de Jong KP, Martens JA (2015) Nanoscale intimacy in bifunctional catalysts for selective conversion of hydrocarbons. Nature 528:245-248

2. Cargnello M, Doan-Nguyen VVT, Gordon TR et al (2013) Control of metal nanocrystal size reveals metal-support interface role for ceria catalysts. Science 341:771-773

3. Prieto G, Zečević J, Friedrich H et al (2013) Towards stable catalysts by controlling collective properties of supported metal nanoparticles. Nat Mater 12:34-39

4. Munnik P, de Jongh PE, de Jong KP (2015) Recent developments in the synthesis of supported catalysts. Chem Rev 115:6687-6718
5. Jia C-J, Schüth F (2011) Colloidal metal nanoparticles as a component of designed catalyst. Phys Chem Chem Phys 13:2457-2487

6. Zaera F (2013) Nanostructured materials for applications in heterogeneous catalysis. Chem Soc Rev 42:2746-2762

7. van Deelen TW, Su H, Sommerdijk NAJM, de Jong KP (2018) Assembly and activation of supported cobalt nanocrystal catalysts for the Fischer-Tropsch synthesis. Chem Commun 54:2530-2533

8. Yin Y, Alivisatos AP (2005) Colloidal nanocrystal synthesis and the organic-inorganic interface. Nature 437:664-670

9. Burda C, Chen X, Narayanan R, El-Sayed MA (2005) Chemistry and properties of nanocrystals of different shapes. Chem Rev 105:1025-1102

10. Puntes VF, Zanchet D, Erdonmez CK, Alivisatos AP (2002) Synthesis of hep-Co nanodisks. J Am Chem Soc 124:12874-12880

11. Comesaña-Hermo M, Ciuculescu D, Li Z-A et al (2012) Stable single domain Co nanodisks: synthesis, structure and magnetism. J Mater Chem 22:8043-8047

12. van Santen RA (2009) Complementary structure sensitive and insensitive catalytic relationships. Acc Chem Res 42:57-66

13. An K, Somorjai GA (2012) Size and shape control of metal nanoparticles for reaction selectivity in catalysis. ChemCatChem $4: 1512-1524$

14. Roldan Cuenya B, Behafarid F (2015) Nanocatalysis: size- and shape-dependent chemisorption and catalytic reactivity. Surf Sci Rep 70:135-187

15. Zaera F (2013) Shape-controlled nanostructures in heterogeneous catalysis. Chemsuschem 6:1797-1820

16. Zhou Y, Li Y, Shen W (2016) Shape engineering of oxide nanoparticles for heterogeneous catalysis. Chem - An Asian J 11:1470-1488

17. Torres Galvis HM, Bitter JH, Khare CB et al (2012) Supported iron nanoparticles as catalysts for sustainable production of lower olefins. Science 335:835-838

18. Chen W, Lin T, Dai Y et al (2017) Recent advances in the investigation of nanoeffects of Fischer-Tropsch catalysts. Catal Today 311:8-22

19. Bezemer GL, Bitter JH, Kuipers HPCE et al (2006) Cobalt particle size effects in the Fischer-Tropsch reaction studied with carbon nanofiber supported catalysts. J Am Chem Soc 128:3956-3964

20. den Breejen JP, Radstake PB, Bezemer GL et al (2009) On the origin of the cobalt particle size effects in Fischer-Tropsch catalysis. J Am Chem Soc 131:7197-7203

21. Prieto G, Martínez A, Concepción P, Moreno-Tost R (2009) Cobalt particle size effects in Fischer-Tropsch synthesis: structural and in situ spectroscopic characterisation on reverse micellesynthesised Co/ITQ-2 model catalysts. J Catal 266:129-144

22. Ducreux O, Rebours B, Lynch J et al (2009) Microstructure of supported cobalt fischer-tropsch catalysts. Oil Gas Sci Technol Rev d'IFP Energies Nouv 64:49-62

23. Sadeqzadeh M, Karaca H, Safonova OV et al (2011) Identification of the active species in the working alumina-supported cobalt catalyst under various conditions of Fischer-Tropsch synthesis. Catal Today 164:62-67

24. Gnanamani MK, Jacobs G, Shafer WD, Davis BH (2013) FischerTropsch synthesis: activity of metallic phases of cobalt supported on silica. Catal Today 215:13-17

25. Liu J-X, Su H-Y, Sun D-P et al (2013) Crystallographic dependence of CO activation on cobalt catalysts: HCP versus FCC. J Am Chem Soc 135:16284-16287

26. Lyu S, Wang L, Zhang J et al (2018) Role of active phase in Fischer-Tropsch synthesis: experimental evidence of $\mathrm{CO}$ activation over single-phase cobalt catalysts. ACS Catal 8:7787-7798

27. Rebmann E, Fongarland P, Lecocq V et al (2017) Measurement of the influence of the microstructure of alumina-supported cobalt catalysts on their activity and selectivity in Fischer-Tropsch 
synthesis by using steady-state and transient kinetics. ChemCatChem 9:2344-2351

28. van Helden P, Ciobîcă IM, Coetzer RLJ (2016) The size-dependent site composition of FCC cobalt nanocrystals. Catal Today 261:48-59

29. Agrawal R, Phatak P, Spanu L (2018) Effect of phase and size on surface sites in cobalt nanoparticles. Catal Today 312:174-180

30. Geerlings JJC, Zonnevylle MC, de Groot CPM (1991) Structure sensitivity of the Fischer-Tropsch reaction on cobalt single crystals. Surf Sci 241:315-324

31. Geerlings JJC, Zonnevylle MC, de Groot CPM (1991) Studies of the Fischer-Tropsch reaction on Co(0001). Surf Sci 241:302-314

32. Qin C, Hou B, Wang J et al (2018) Crystal-plane-dependent Fischer-Tropsch performance of cobalt catalysts. ACS Catal 8:9447-9455

33. Oosterbeek H (2007) Bridging the pressure and material gap in heterogeneous catalysis: cobalt Fischer-Tropsch catalysts from surface science to industrial application. Phys Chem Chem Phys 9:3570-3576

34. Navarro V, van Spronsen MA, Frenken JWM (2016) In situ observation of self-assembled hydrocarbon Fischer-Tropsch products on a cobalt catalyst. Nat Chem 8:929-934

35. Venvik HJ, Borg A, Berg C (1998) Formation of the CO-induced $(3 \times 1)$ surface structure on $\operatorname{Co}(11-20)$ studied by STM. Surf Sci 397:322-332

36. Strømsheim MD, Svenum I-H, Farstad MH et al (2018) Effects of $\mathrm{K}$ adsorption on the CO-induced restructuring of $\mathrm{Co}(11-20)$. Catal Today 299:37-46

37. Schulz H, Nie Z, Ousmanov F (2002) Construction of the FischerTropsch regime with cobalt catalysts. Catal Today 71:351-360

38. Wilson J, de Groot CPM (1995) Atomic-scale restructuring in high-pressure catalysis. J Phys Chem 99:7860-7866

39. Banerjee A, Navarro V, Frenken JWM et al (2016) Shape and size of cobalt nanoislands formed spontaneously on cobalt terraces during Fischer-Tropsch synthesis. J Phys Chem Lett 7:1996-2001

40. Høydalsvik K, Fløystad JB, Voronov A et al (2014) Morphology changes of Co catalyst nanoparticles at the onset of FischerTropsch synthesis. J Phys Chem C 118:2399-2407

41. Weststrate CJ, Kizilkaya AC, Rossen ETR et al (2012) Atomic and polymeric carbon on $\mathrm{Co}(0001)$ : surface reconstruction, graphene formation, and catalyst poisoning. J Phys Chem C 116:11575-11583

42. Zhong L, Yu F, An Y et al (2016) Cobalt carbide nanoprisms for direct production of lower olefins from syngas. Nature 538:84-87

43. Scariot M, Silva DO, Scholten JD et al (2008) Cobalt nanocubes in ionic liquids: synthesis and properties. Angew Chem Int Ed 47:9075-9078

44. Ba R, Zhao Y, Yu L et al (2015) Synthesis of Co-based bimetallic nanocrystals with one-dimensional structure for selective control on syngas conversion. Nanoscale 7:12365-12371

45. Wen C, Dunbar D, Zhang X et al (2014) Self-healing catalysts: $\mathrm{Co}_{3} \mathrm{O}_{4}$ nanorods for Fischer-Tropsch synthesis. Chem Commun 50:4575-4578

46. Harmel J, Berliet A, Dembélé K et al (2018) A seed-mediated approach for the preparation of modified heterogeneous catalysts. ChemCatChem 10:1614-1619
47. Harmel J, Peres L, Estrader M et al (2018) hcp-Co nanowires grown on metallic foams as catalysts for the Fischer-Tropsch synthesis. Angew Chem Int Ed 57:10579-10583

48. Puntes VF, Krishnan KM, Alivisatos AP (2001) Colloidal nanocrystal shape and size control: the case of cobalt. Science 291:2115-2117

49. van Deelen TW, Nijhuis JJ, Krans NA et al (2018) Preparation of cobalt nanocrystals supported on metal oxides to study particle growth in Fischer-Tropsch catalysts. ACS Catal 8:10581-10589

50. Reuel RC, Bartholomew CH (1984) The stoichiometries of $\mathrm{H}_{2}$ and $\mathrm{CO}$ adsorptions on cobalt: effects of support and preparation. J Catal 85:63-77

51. Leijten ZJWA, Keizer ADA, de With G, Friedrich H (2017) Quantitative analysis of electron beam damage in organic thin films. $J$ Phys Chem C 121:10552-10561

52. Kremer JR, Mastronarde DN, McIntosh JR (1996) Computer visualization of three-dimensional image data using IMOD. J Struct Biol 116:71-76

53. Schindelin J, Arganda-Carreras I, Frise E et al (2012) Fiji: an open-source platform for biological-image analysis. Nat Methods 9:676-682

54. Cats KH, Weckhuysen BM (2016) Combined operando X-ray diffraction/raman spectroscopy of catalytic solids in the laboratory: the $\mathrm{Co} / \mathrm{TiO}_{2}$ Fischer-Tropsch synthesis catalyst showcase. ChemCatChem 8:1531-1542

55. Xie J, Paalanen PP, van Deelen TW et al (2019) Promoted cobalt metal catalysts suitable for the production of lower olefins from natural gas. Nat Commun 10:167

56. Puntes VF, Krishnan K, Alivisatos AP (2002) Synthesis of colloidal cobalt nanoparticles with controlled size and shapes. Top Catal 19:145-148

57. Munnik P, de Jongh PE, de Jong KP (2014) Control and impact of the nanoscale distribution of supported cobalt particles used in Fischer-Tropsch catalysis. J Am Chem Soc 136:7333-7340

58. Eschemann TO, Lamme WS, Manchester RL et al (2015) Effect of support surface treatment on the synthesis, structure, and performance of Co/CNT Fischer-Tropsch catalysts. J Catal 328:130-138

59. Sławiński WA, Zacharaki E, Fjellvåg H, Sjåstad AO (2018) Structural arrangement in close-packed cobalt polytypes. Cryst Growth Des 18:2316-2325

60. Andreev AS, d'Espinose de Lacaillerie J-B, Lapina OB, Gerashenko A (2015) Thermal stability and hcp-fcc allotropic transformation in supported Co metal catalysts probed near operando by ferromagnetic NMR. Phys Chem Chem Phys 17:14598-14604

61. Srinivasan R, De Angelis RJ, Reucroft PJ et al (1989) Structural characterization of cobalt catalysts on a silica support. J Catal 116:144-163

62. Garces LJ, Hincapie B, Zerger R, Suib SL (2015) The effect of temperature and support on the reduction of cobalt oxide: an in situ X-ray diffraction study. J Phys Chem C 119:5484-5490

Publisher's Note Springer Nature remains neutral with regard to jurisdictional claims in published maps and institutional affiliations. 